\title{
Modeling the Cray Memory Scheduler
}

Keith L. Wickham

Gerald L. Litteer

Published April 1992

Idaho National Engineering Laboratory EG\& G Idaho, Inc. Idaho Falls, Idaho 83415

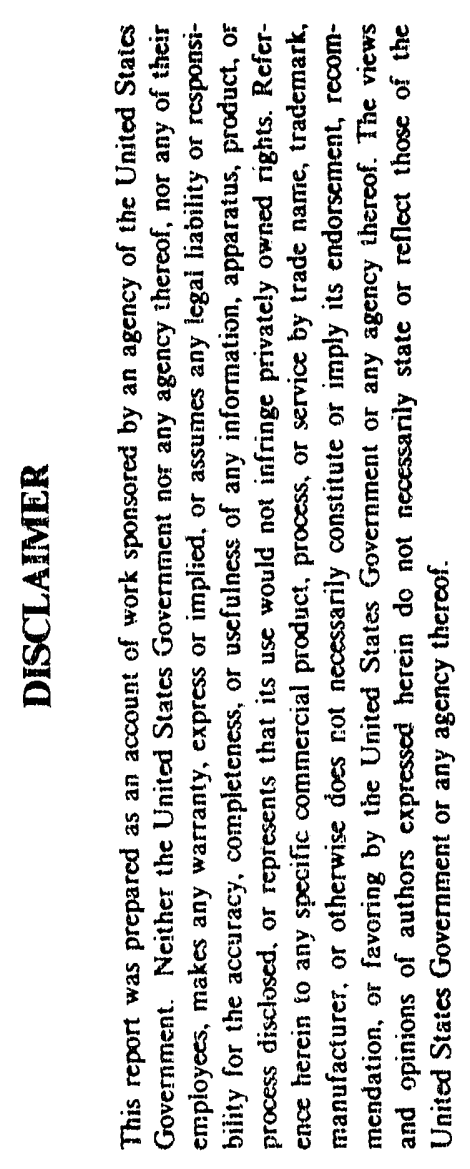

Prepared for the

U.S. Department of Energy Through the

EG\&G Idaho LDRD Program Under DOE Idaho Field Office Contract DE-AC07-761D01570

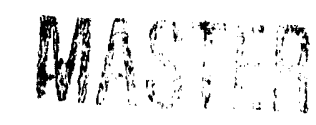




\begin{abstract}
This report documents the results of a project to evaluate low cost modeling and simulation tools when applied to modeling the Cray memory scheduler. The specific tool used is described and the basics of the memory scheduler are covered. Results of simulations using the model are discussed and a favorable recommendation is made to make more use of this inexpensive technology.
\end{abstract}




\section{CONTENTS}

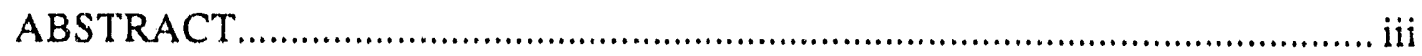

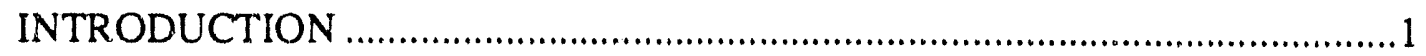

THE MODELING PACKAGE ..............................................................

THE CRAY MEMORY SCHEDULER ...................................................

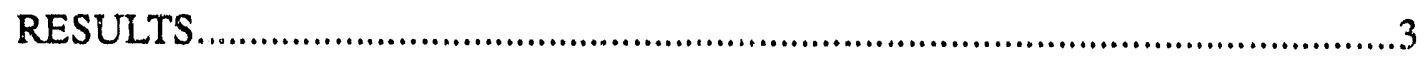

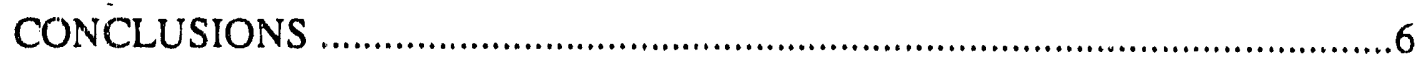




\title{
Modeling the Cray Memory Scheduler
}

\author{
INTRODUCTION
}

The availability of low cost modeling and simulation tools makes it possible to investigate problems that have previously been ignored because of the cost of writing custom models in FORTRAN or C code. This report documents the results of a project to evaluate a modeling tool and to apply it to the problem of tuning the system parameters that control memory scheduling on a Cray ${ }^{\text {a } X M P / 216}$ computer at the INEL.

\section{THE MODELING PACKAGE}

The modeling package used for this work was "ithink" from High Performance Systems, Inc. The package runs on an Apple Macintosh computer and uses an interactive, graphical interface that is comrnon on this brand of computer. The package can be thought of as an advanced spread sheet. It is capable of handling typical financial models; however, the advanced features are well suited to other problems, especially simulations.

"ithink" turns a normal spreadsheet inside out. The data interconnections are shown graphically on the outside and the numbers are hidden inside. The reasoning behind this approach is that the relationships, not the numbers, deserve primary attention. Instead of the traditional spread sheet with rigid rows and columns and meaningless names such as $\mathrm{A} 1$ or $\mathrm{B} 3$, there are different symbols for each type of tool freely placed on the screen with connecting arrows and meaningful names such as "cost per unit" or "total time". A second difference is how time is handled. With a traditional spread sheet, a model involving twelve months requires twelve columns, which are often duplicates of each other. With "ithink", the model is defined for one period and then a run parameter is set for the number of periods. The result for any period may be seen in the output that is produced.

While the numbers are hidden, they are easily accessed. Values or equations may be seen and changed by clicking the mouse on each tool symbol. A popup windo $N$ brings the information up. Results of a sirnulation can be organized in spread sheet-like tables or visualized in graphical format. For simulation work, the graphical output was very useful. The graph is drawn as the simulation runs, allowing instant gratification, or the ability to stop a simulation that has gone awry.

The feature of the "ithink" package that allowed it to model the Cray memory scheduler was a tool called a reservoir. This is essentially an accumulator with an inflow and an outflow. It allows feedback loops to be modeled without creating the circular relationships that spread sheets typically disallow. Defining the flow in or out to depend on the current value of the reservoir is accept.

a. Mention of specific products and/or manufactures in this document implies neither endorsement or preference, nor disapproval by the U.S. Government, any of its agencies, or EG\&G Idaho, Inc., of the use of a specific product for any purpose. 
able. As the example also implies, the "ithink" package can model problems involving first-order differential equations.

The "ithink" package has several other tools that are not found in ordinary spreadsheets. For modeling discrete processes it has a conveyor for inserting delays, a queue for waiting in line, and an oven for batch processing. The functions that can be used in equations include financial (present value, future value, etc.), mathernatical (mean, square root, etc.), trigonometric (sine, cosine, etc.), and statistical (random, Poisson distributed random, etc.).

The documentation included with the "ithink" package is different than what normally comes with a software package. There is no alphabetical reference of cryptic commands. Instructions on running the package are presented in tutorial fashion in a manual that is more like a best seller. It contains numerous examples of non-financial models that are presented in a thought provoking manner. The authors use the term "System Thinking" to describe their view of modeling. There are even chapters on how to generically approach the modeling process. The examples contain many good ideas that can be incorporated into specific models.

The "ithink" package does have limitations that should be considered when applying it to modeling problems. The size of our problem is about the maximum that is practical. The model used here takes 9 pages to print the graphical representation and another 9 pages to print the underlying equations. It requires 1.5 Mbytes on the Macintosh to execute. Another limitation is that time is the oniy loop within the program. This was partially overcome by using a reservoir as a counter to establish steps for complex operations. This required the model to use time steps representing one tenth of a second, which forced a test to be placed on many operations to know when a full second had passed.

\section{THE CRAY MEMORY SCHEDULER}

The architecture of the Cray computer requires an entire process to be in memory before it can be allowed to run. The process does not have to start at the same physical address each time, but it will always be in one contiguous block. There is no virtual addressing or demand paging as is found in many other brands of computers. This creates a need for a memory scheduler to work in conjunction with the memory swapper to determine which processes should be in memory at any instant in time and which ones should be swapped to disk. If a process is not eligible to run because it is waiting for some event to occur, then it becomes a prime candidate to be swapped out if more memory is needed by an another process that is ready to run. When there are more processes eligible to run than memory to hold them, the memory scheduler decides who will be swapped in or out, based on a swap priority.

The swap priority of a process is a real number calculated by the memory scheduler using several factors. The system parameters that relate to swap priority are generally arranged in pairs. The pairs allow the swap-in and swap-out priority to be calculated differently. When the highest swapin priority of an out-of-memory process exceeds the lowest swap-out priority of an in-rnemory process, a swap is made. The final scheduler model for this research included five pairs and one independent parameter. The parameters are a pair of memory factors, a pair of time factors, a pair of "nice" factors, a pair of priority factors, a pair of share factors, and a single parameter called fit 
boost. The appropriate factor is multiplied by the corresponding process value of memory, time, nice, priority, and share. The results are added together and then, if the process is in memory, the fit boost is added to the total. In simple terms, the memory factors determine a base value and the time factor decays this until swapping occurs. The other factors fine tune the basic algorithm.

There are twenty-one system parameters relating to swap priority. Processes requiring large amounts of memory or using more than a certain amount of cpu time are called "hogs". The hog process parameters were not modeled, but these are easily set independently based on things such as physical memory size. The model did not include parameters based on minimum memory residency or swap thrash rates. These are generally a complementary set to the parameters chosen and are not intended to be used at the same time. The model was limited to 5 processes being eligible to run because of limitations of the modeling package. Admittedly, the model does not contain all the features of the actual process, but it included those needed to tune the scheduler.

The Cray also runs a "Fair Share" scheduler that attempts to keep the cpu usage equal for processes that have the same number of shares in the system. We are running the minimum version of Fair Share in which all users have the same number of shares and the time deray factors are set very short. Basically Fair Share accumulates a share priority based on the amount of cpu time a process received. This value is decayed periodically by an amount based on the nice value of the process. The share priorities of all the processes are then normalized to yroduce an execution priority for each process. The more cpu time a process has received, the lower its execution priority will be in the next interval, relative to the other processes. Conversely, the less cpu time a process has received, the higher its execution priority will be. The Fair Share scheduler had to be incorporated into the model because it causes an interaction between nice, share, and priority.

\section{RESULTS}

A series of cases was run with different system parameters. The goal was to learn the range of each parameter and to find the best combination for our system and load. Using hindsight, the range of the values is easily seen. For example, since priority ranges from 0 to 999 and nice ranges from 0 to 39 , the nice factors will have to be larger to have the same effect. Since priority and nice are inversely proportional, i.e., la"ge numbers mean less, the factors need to be negative to tune the scheduler in the desired direction. The complication that makes a model necessary is that while nice is fixed for a process, both share and priority are changed by the Fair Share scheduler as the process runs. Also, because these factors are inter-related, only one should be used to control scheduling and the others set to zero. The exercise of modeling gave us a much better understanding of how the scheduler really works. After many different cases were tried, we concluded that the system parameters currently in use on the Cray are very close to optimum for our work load.

We did find one change that we plan to make on the actual machine. The fit boost parameter is currently set to zero. This is the amount that is added to an in-memory process as part of its swap priority calculation. The recommendation from Cray is to set this to -0.5 to provide a small pressure for in-memory processes to swap out. With our understanding of the scheduler, we see that this factor has more effect on small processes. Applying our knowledge of the system load, we find many small processes are used for short time periods. By setting the fit boost to +1.0 we are 
setting a minium memory residency that may allow the small process to complete its task without being swapped.

There are three cases that will be discussed in more detail. Case 1 is five processes competing equally for memory. Case 2 is two large and three small processes showing hog interactions. Case 3 is 1 large and 4 small processes showing a typical load.

Case 1 was created to test our model and establish a baseline for changes to system parameters in future cases. Five equal processes were created and the memory size set, so that only three could be in memory at one time. The current Cray system parameters were used which have zero in the priority factor, nice factor, and fit boost. A plot of the swap priority values uver time is shown in Figure 1. Periodically the process with the most time in memory swapped with the process with the most time out of memory. It is also interesting that while the initial values for time were poorly chosen, the model rapidly developed a uniform time spacing between swapping. The share calculation yielded curves similar to those provided by Cray from actual measurements. The total cpu time received by the processes was approximately the same at the end of the run.

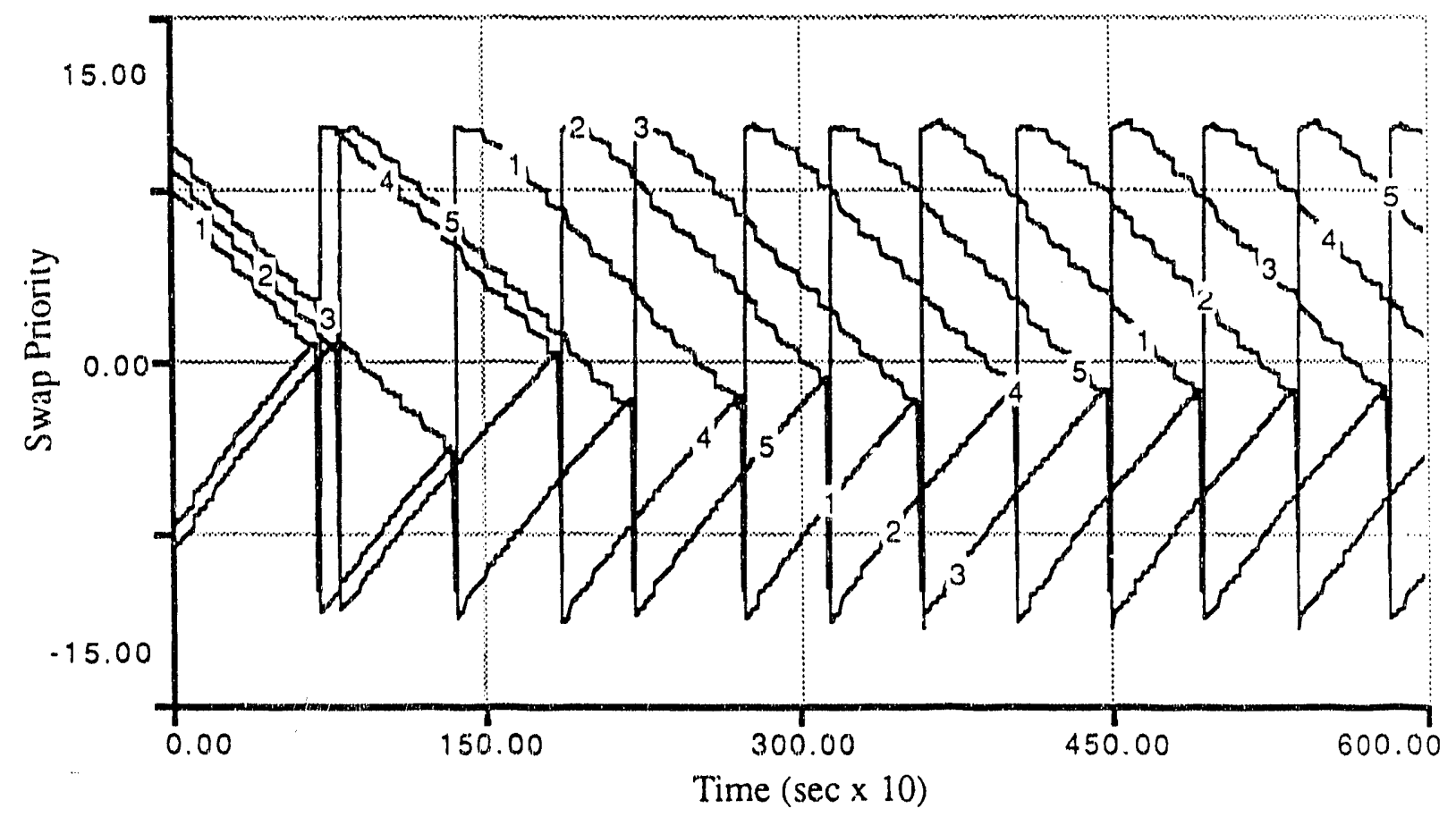

Figure 1. Swap Priority for Five Equal Processes.

Case 2 used two large processes at low priority with three small pocesses at normal priority. The memory size was set so that one large process and one small process, or all three small processes, could be in memory at one time. A plot of the swap priority values over time is shown in Figure 2. The result is similar to what would happen in the case of the "hogs" The large processes competed with each other and the small processes competed with each other. The total cpu time received by the processes reflected the difference in priority. Equal priority processes receive approximately the same amount of time, but the higher priority group received more time than the lower priority group. 


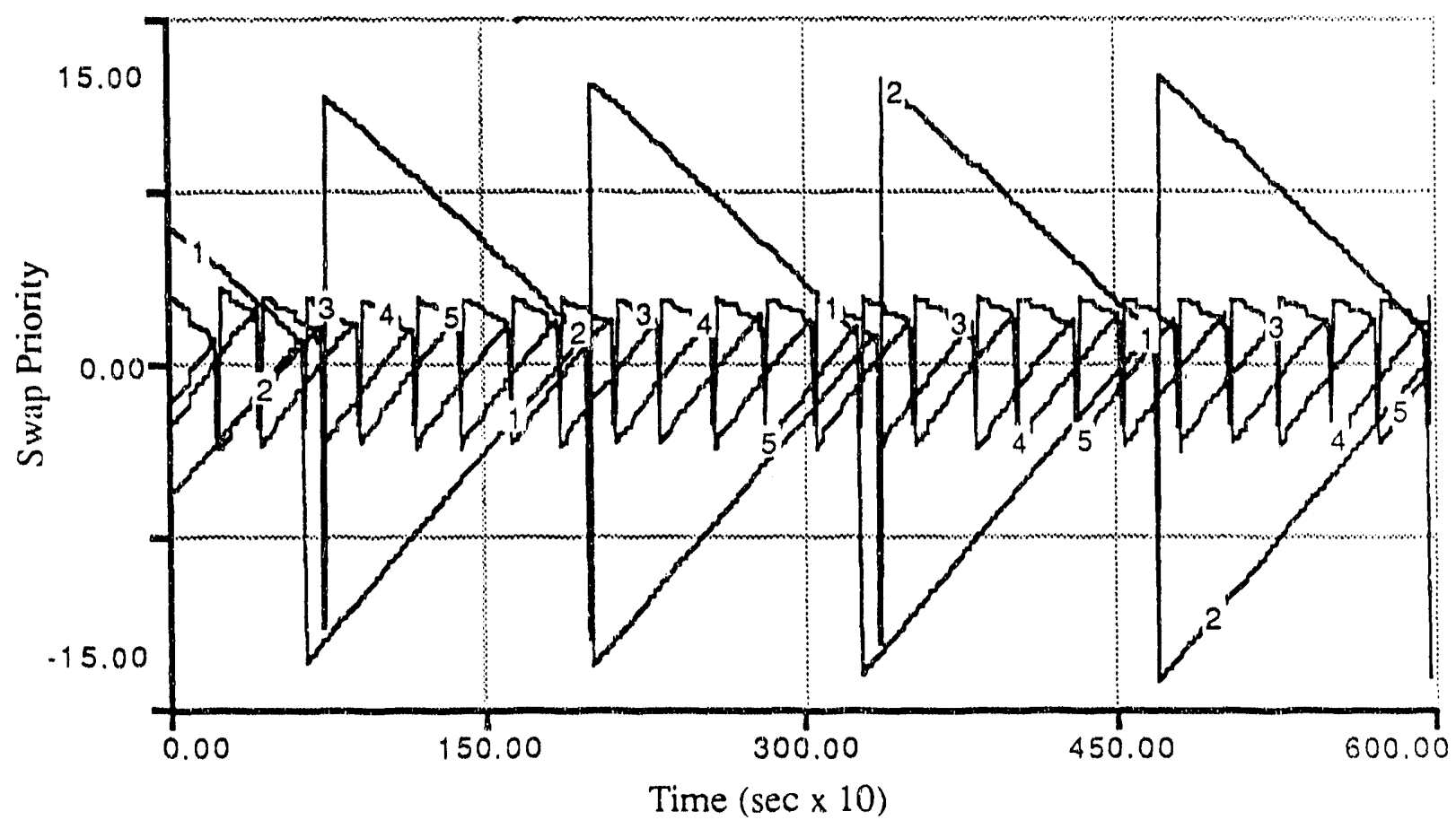

Figure 2. Swap Priority for Two Large and Three Small Processes.

Case 3 represented our typical load consisting of one large process at low priority and four small processes at normal priority. The memory size was set to allow the large process and one small process, or all four small processes to be in memory at one time. A plot of the swap priority is shown in Figure 3. Several interesting things are shown in this case. The large process spent more time in memory than out of memory. When the large process swapped into memory, it took time for the small processes to develop a uniform time spacing between swaps. There was a noticeable difference in their cpu tirne accumulations because of the interference by the large process. The effect of the share factor was very noticeable in the small process when the large process was in memory. The small processes had a higher execution priority than the large process and accumulated shares rapicly. The upper slope of their plots became flatter because of the share factor which causes them to stay in memory ionger. This factor keeps processes exhibiting high usage in memory to insure the cpu is fully utilized.

The model was also used for cases that were not even considered when the project started. The rate structure used for computer charges is based on the user specified value for the process nice parameter. Several cases were run with different combinations of process nice values to see if the difference in execution time was fairly reflected in the rate structure. The answer turned out to depend heavily on system load. If all processes specified a high priority (low numerical nice value) they all paid a high price without any gain. Conversely, if all processes specified a low priority, they competed equally but paid a low price. In a uniform mix of priorities, the rate structure penalizes high priority processes and rewards low priority processes. This was the intent in the design of the rate structure. 


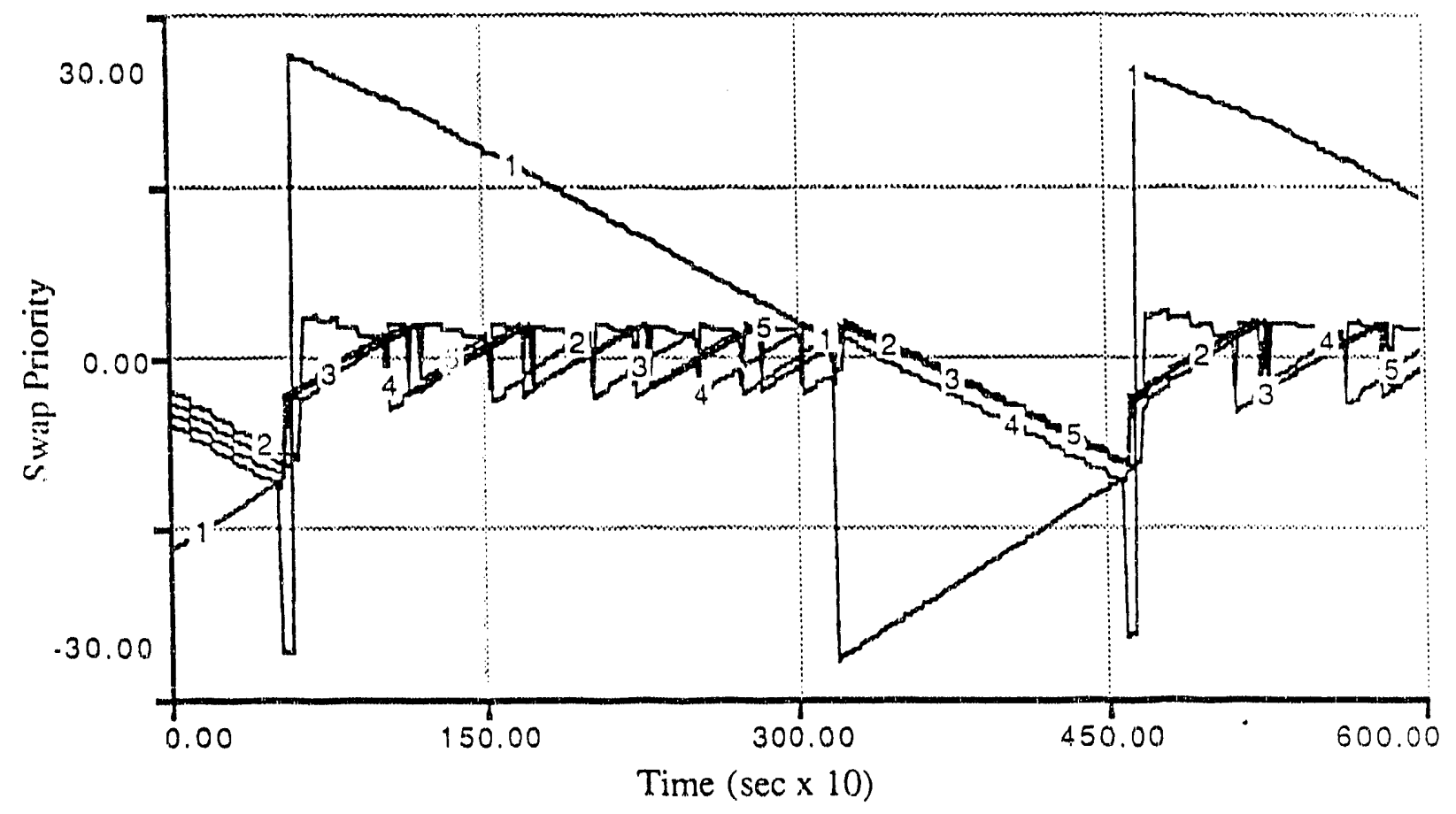

Figure 3. Swap Priority for Typical Load

A second version of the model was created to investigate the effect of the Fair Share scheduler parameters. The time decay values were increased to see what effect this would have on memory scheduling. With all processes equal, the Fair Share scheduler had no effect; however, when processes with different initial share and nice values were !sed, the effect of Fair Share became more pronounced. High "nice" processes did not decay their share value as rapidly and consequently remained high share processes. Processes with high share values received less priority for $\mathrm{cpu}$ time even when they were in memory. The larger time values slowed the decay of share values so that prior history became more important. The overall effect was to penalize processes that has accumulated high shares in the past and to reward processes that had low share values. When we start to use all the features of the Fair Share scheduler, processes will be grouped so that usage by other members of the group effects the execution prioriiy. This model will be useful to help select the correct time decay values and adjust the share factors used by the memory scheduler. When dealing with these kinds of interdependencies, a model of the system is a very valuable tool.

\section{CONCLUSIONS}

The availability of low cost modeling and simulation tools allows investigation in areas that would not otherwise be cost effective. Using these tools, the modeler can concentrate on the problem instead of the software. The exercise of creating a model also produces a better understanding of the process. Relationships that are not clearly understood must be researched anc specified precisely in the model. Failures of the model to reflect reality forces one to think about the assumptions used in the model. This also can provide insight on improving the process. This project has been beneficial in expanding our knowledge of new modeling tools and in solving our specific 
problem. We have already applied modeling to two related problems that were not envisioned when the project started. We plan to incorporate the modeling technique for future releases of the Cray operating system, expanding the model and our concept of the scheduling algorithms as we go forward. This approach to problem solving is clearly superior to the trial and error method that was used previously. 

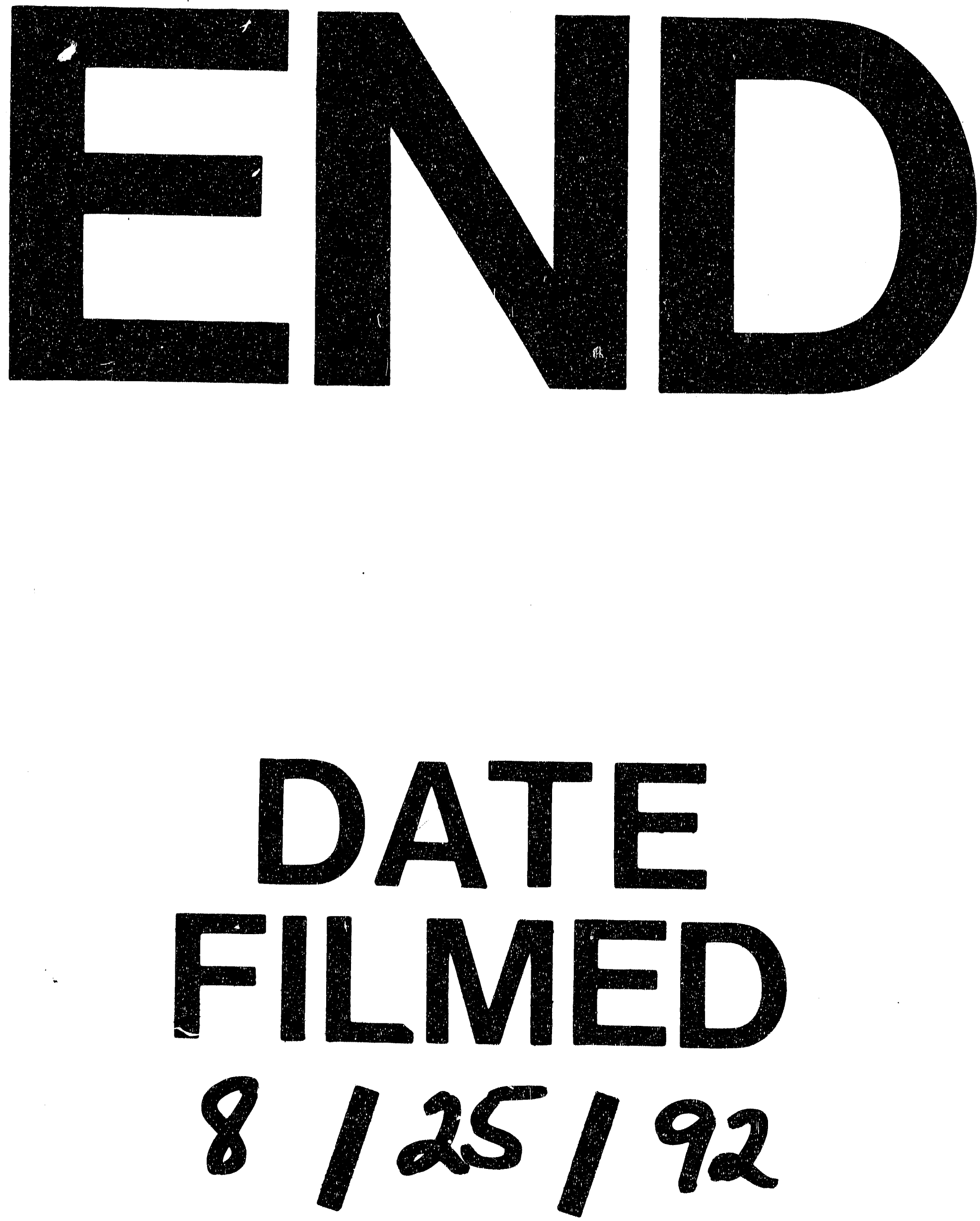
